\title{
Debye-Series Analysis of the First-Order Rainbow Produced in Scattering of a Diagonally Incident Plane Wave by a Circular Cylinder
}

\author{
James A. Lock \\ Cleveland State University, j.lock@csuohio.edu \\ Charles L. Adler \\ Follow this and additional works at: https://engagedscholarship.csuohio.edu/sciphysics_facpub \\ Part of the Physics Commons \\ How does access to this work benefit you? Let us know!
}

\section{Publisher's Statement}

This paper was published in Journal of the Optical Society of America A: Optics Image Science and Vision and is made available as an electronic reprint with the permission of OSA. The paper can be found at the following URL on the OSA website: http://www.opticsinfobase.org/josaa/ abstract.cfm?URI=josaa-14-6-1316. Systematic or multiple reproduction or distribution to multiple locations via electronic or other means is prohibited and is subject to penalties under law.

\section{Original Citation}

Lock, James A. and C. L. Adler. "Debye-Series Analysis of the First-Order Rainbow Produced in Scattering of a Diagonally Incident Plane Wave by a Circular Cylinder." Journal of the Optical Society of America A: Optics Image Science and Vision 14 (1997): 1316-1328.

\section{Repository Citation}

Lock, James A. and Adler, Charles L., "Debye-Series Analysis of the First-Order Rainbow Produced in Scattering of a Diagonally Incident Plane Wave by a Circular Cylinder" (1997). Physics Faculty Publications. 62.

https://engagedscholarship.csuohio.edu/sciphysics_facpub/62

This Article is brought to you for free and open access by the Physics Department at EngagedScholarship@CSU. It has been accepted for inclusion in Physics Faculty Publications by an authorized administrator of EngagedScholarship@CSU. For more information, please contact library.es@csuohio.edu. 


\title{
Debye-series analysis of the first-order rainbow produced in scattering of a diagonally incident plane wave by a circular cylinder
}

\author{
James A. Lock and C. L. Adler \\ Department of Physics, Cleveland State University, Cleveland, Ohio 44115
}

Received July 12, 1996; accepted October 28, 1996; revised manuscript received December 3, 1996

\begin{abstract}
We derive the Debye-series expansion of the partial-wave scattering and interior amplitudes for the interaction of a diagonally incident beam of arbitrary profile with an infinitely long homogeneous dielectric circular cylinder. We then discuss the physical interpretation of the various terms of the series. We also consider the one-internal-reflection Debye-series terms for diagonal plane-wave incidence and examine the first-order rainbow extinction transition as a function of the tilt angle of the incident plane wave. We experimentally observe the first-order rainbow extinction transition and compare our observations with the Debye-series predictions. (C) 1997 Optical Society of America [S0740-3232(97)01906-6]
\end{abstract}

\section{INTRODUCTION}

The electromagnetic boundary-value problem of a diagonally incident plane wave scattered by an infinitely long homogeneous dielectric circular cylinder was solved exactly by Wait ${ }^{1}$ in 1955 . In his solution the incident, scattered, and interior scalar radiation potentials ${ }^{2}$ were expanded as an infinite series of partial waves. The partial-wave scattering and interior amplitudes were obtained by requiring continuity of the tangential components of the electric and magnetic fields at the surface of the cylinder. The amplitudes produced by this procedure are complicated combinations of Bessel and Hankel functions, thus obscuring the physical interpretation of the various features of the scattered fields.

Nonetheless, much progress has been made in understanding diagonal incidence/cylinder scattering in intuitive and physical terms. From the outset it was recognized that the diagonal beam/cylinder geometry causes a mixture of polarization-preserving and cross-polarized scattering, ${ }^{1}$ a feature absent in plane-wave scattering by a sphere or by a cylinder at normal incidence. Kerker et $a l .^{3}$ have shown that the scattering amplitudes for the two cross-polarized channels differ only in sign. Cohen and Acquista ${ }^{4}$ showed that the result of Kerker et al. was a consequence of time reversal invariance, and they also discussed the restrictions that energy conservation place on the partial-wave scattering amplitudes. Takano and Tanaka ${ }^{5}$ derived the ray theory expression for the diagonal incidence/cylinder scattered intensity. They also discussed a number of features of the scattered intensity in this context.

The purpose of this paper is to connect the wave and ray descriptions of diagonal incidence/cylinder scattering by obtaining the Debye-series decomposition of the partial-wave scattering and interior amplitudes. The terms of the Debye series, summed over partial waves, correspond to diffraction plus the wave analogies to the fundamental scattering processes of ray theory, i.e., re- flection, transmission, and transmission following a number of internal reflections. In the short-wavelength limit, many partial waves contribute to the scattered fields. As a result, the sum over partial waves for each term of the Debye series may be converted into an integral over an associated impact parameter. The stationary-phase approximation of the integral produces the scattered field of ray theory, and the stationary-phase location is associated with the trajectory of the light ray. ${ }^{6-9}$

The body of this paper is organized as follows. In Section 2 we briefly describe the geometry of a beam with arbitrary profile and that is diagonally incident on an infinitely long circular cylinder. We also give the formulas for the scattered and interior electric and magnetic fields in wave theory. In Section 3 we derive the 16 partialwave reflection and transmission Fresnel coefficients that occur for this geometry. In Section 4 we write the partial-wave scattering and interior amplitudes as an infinite series of interactions of the partial wave with the cylinder surface, with each term in the series being a particular combination of these Fresnel coefficients. In Section 5 we apply the Debye-series formalism to the firstorder rainbow produced when the incident beam is a plane wave, and we examine the rainbow extinction transition. In Section 6 we observe the first-order rainbow extinction transition experimentally and compare our observations with the Debye-series predictions. In Section 7 we present our conclusions.

\section{PARTIAL-WAVE SCATTERING AND INTERIOR AMPLITUDES}

We consider an infinitely long homogeneous circular cylinder of radius $a$ and refractive index $n$ whose axis coincides with the $z$ axis of a rectangular coordinate system. A monochromatic beam (e.g., a plane wave or a focused Gaussian beam) with wave number $k=2 \pi / \lambda$ and propagation direction $\hat{k}_{\text {inc }}$ in the $x-z$ plane and making an angle $\xi$ with the $x$ axis, i.e., 


$$
\hat{k}_{\text {inc }}=(\cos \xi) \hat{u}_{x}-(\sin \xi) \hat{u}_{z},
$$

strikes the cylinder and is scattered. The incident beam, the scattered wave, and the interior wave are $\epsilon$ polarized if their electric fields lie in the horizontal $(x-y)$ plane, and they are $\mu$ polarized if their magnetic fields lie in the horizontal plane.

The partial-wave expansion of the fields in cylindrical coordinates ${ }^{10}$ is parameterized by two indices $l$ and $h$. The discrete index $l$ is an integer with $-\infty<l<\infty$ and is called the partial wave number. The continuous index $h$ with $-\infty<h<\infty$ describes the profile of the incident beam. For a plane wave, $h$ assumes the single value $h$ $=-\sin \xi$, in accordance with Eq. (1). For a focused Gaussian beam, a range of $h$ values that depend on the width of the beam and are centered about $h=-\sin \xi$ is required. The weighting coefficients in the partial-wave expansion of the $\epsilon$-polarized portion of the incident beam are $A_{l}(h)$. The coefficients in the partial-wave expansion of the $\mu$-polarized portion of the beam are $B_{l}(h)$. Together they are termed the beam-shape coefficients.

For the diagonally incident beam/cylinder geometry, the partial-wave scattering and interior amplitudes for $\epsilon$-polarization-preserving scattering ( $\epsilon$ incident goes to $\epsilon$ scattered or interior) are $\mathrm{e}^{10}$

$$
\begin{aligned}
& a_{l}(h)=\frac{U_{2} W_{1}-n U_{3} W_{3}}{D}, \\
& c_{l}(h)=\frac{-2 i n x W_{1}}{\pi y^{2} D},
\end{aligned}
$$

respectively, where

$$
\begin{aligned}
x & \equiv k a\left(1-h^{2}\right)^{1 / 2}, \quad y \equiv n k a\left(1-h^{2} / n^{2}\right)^{1 / 2}, \\
U_{1} & =\frac{n^{2} x}{y} J_{l}(x) J_{l}^{\prime}(y)-J_{l}^{\prime}(x) J_{l}(y), \\
U_{2} & =\frac{n x}{y} J_{l}(x) J_{l}^{\prime}(y)-n J_{l}^{\prime}(x) J_{l}(y), \\
U_{3} & =\frac{h l\left(y^{2}-x^{2}\right)}{x y^{2}} J_{l}(x) J_{l}(y), \\
W_{1} & =\frac{n^{2} x}{y} H_{l}^{(1)}(x) J_{l}^{\prime}(y)-H_{l}^{(1)^{\prime}}(x) J_{l}(y), \\
W_{2} & =\frac{n x}{y} H_{l}^{(1)}(x) J_{l}^{\prime}(y)-n H_{l}^{(1)^{\prime}}(x) J_{l}(y), \\
W_{3} & =\frac{h l\left(y^{2}-x^{2}\right)}{x y^{2}} H_{l}^{(1)}(x) J_{l}(y), \\
D & =W_{1} W_{2}-n W_{3}^{2},
\end{aligned}
$$

with the $J_{l}$ being Bessel functions and the $H_{l}^{(1)}$ being Hankel functions of the first type. Similarly, the partialwave scattering and interior amplitudes for $\mu$-polarization-preserving scattering ( $\mu$ incident goes to $\mu$ scattered or interior) are

$$
\begin{aligned}
& b_{l}(h)=\frac{U_{1} W_{2}-n U_{3} W_{3}}{D}, \\
& d_{l}(h)=\frac{-2 i n x W_{2}}{\pi y^{2} D},
\end{aligned}
$$

respectively. The partial-wave scattering and interior amplitudes for cross-polarized scattering $(\epsilon$ incident goes to $\mu$ scattered or interior, or $\mu$ incident goes to $\epsilon$ scattered or interior) are

$$
\begin{aligned}
& q_{l}(h)=\frac{2 n \ln \left(y^{2}-x^{2}\right) J_{l}^{2}(y)}{\pi x^{2} y^{2} D}, \\
& p_{l}(h)=\frac{-2 n x W_{3}}{\pi y^{2} D}
\end{aligned}
$$

respectively.

The scattered electric and magnetic fields in the far zone are constructed from the partial-wave scattering amplitudes according to ${ }^{10}$

$$
\begin{aligned}
\lim _{r \rightarrow \infty} \mathbf{E}_{\text {scatt }}(r, \theta, z)= & \frac{E_{0}}{\cos \xi}\left(\frac{2}{\pi k r}\right)^{1 / 2}[\exp (-i \pi / 4)] \\
& \times\left(T_{5} \hat{u}_{r}-T_{2} \hat{u}_{\theta}-T_{3} \hat{u}_{z}\right), \\
\lim _{r \rightarrow \infty} \mathbf{B}_{\text {scatt }}(r, \theta, z)= & \frac{E_{0} / c}{\cos \xi}\left(\frac{2}{\pi k r}\right)^{1 / 2}[\exp (-i \pi / 4)] \\
& \times\left(T_{6} \hat{u}_{r}+T_{1} \hat{u}_{\theta}-T_{4} \hat{u}_{z}\right),
\end{aligned}
$$

where $E_{0}$ is a measure of the peak electric-field strength of the incident beam and the summed and integrated scattering amplitudes $T_{i}$ for $1 \leqslant i \leqslant 6$ are

$$
\begin{aligned}
& T_{1}=\int_{-\infty}^{\infty} \mathrm{d} h \sum_{l=-\infty}^{\infty}\left(1-h^{2}\right)^{1 / 4} \beta_{l}(h) \Phi(h, l), \\
& T_{2}=\int_{-\infty}^{\infty} \mathrm{d} h \sum_{l=-\infty}^{\infty}\left(1-h^{2}\right)^{1 / 4} \alpha_{l}(h) \Phi(h, l), \\
& T_{3}=\int_{-\infty}^{\infty} \mathrm{d} h \sum_{l=-\infty}^{\infty}\left(1-h^{2}\right)^{3 / 4} \beta_{l}(h) \Phi(h, l), \\
& T_{4}=\int_{-\infty}^{\infty} \mathrm{d} h \sum_{l=-\infty}^{\infty}\left(1-h^{2}\right)^{3 / 4} \alpha_{l}(h) \Phi(h, l), \\
& T_{5}=\int_{-\infty}^{\infty} \mathrm{d} h \sum_{l=-\infty}^{\infty} h\left(1-h^{2}\right)^{1 / 4} \beta_{l}(h) \Phi(h, l), \\
& T_{6}=\int_{-\infty}^{\infty} \mathrm{d} h \sum_{l=-\infty}^{\infty} h\left(1-h^{2}\right)^{1 / 4} \alpha_{l}(h) \Phi(h, l) .
\end{aligned}
$$

The phase $\Phi(h, l)$ in the integrands of Eqs. (13) is

$$
\Phi(h, l)=\exp \left[i k r\left(1-h^{2}\right)^{1 / 2}\right] \exp (i k h z) \exp (i l \theta),
$$

and the dependence of the scattered fields on the incidentbeam profile is 


$$
\begin{aligned}
& \alpha_{l}(h)=a_{l}(h) A_{l}(h)+q_{l}(h) B_{l}(h), \\
& \beta_{l}(h)=-q_{l}(h) A_{l}(h)+b_{l}(h) B_{l}(h) .
\end{aligned}
$$

For an incident plane wave, the summed and integrated scattering amplitudes $T_{i}$ simplify substantially, since $A_{l}(h)$ and $B_{l}(h)$ are $\delta$ functions in $h$, thus allowing the $h$ integrals in Eqs. (13) to be performed exactly. This is pursued further in Section 5. For an incident focused Gaussian beam, the scattering amplitudes again simplify substantially, since the $h$ integrals are sharply peaked about $h=-\sin \xi$ and thus may be approximated in the far zone by the stationary-phase method. ${ }^{10}$

The interior electric field is ${ }^{11}$

$$
\begin{aligned}
\mathbf{E}_{\text {interior }}(r, \theta, z)= & \frac{E_{0}}{\cos \xi} \int_{-\infty}^{\infty} \mathrm{d} h \sum_{l=-\infty}^{\infty} i^{l+1} \\
& \times \exp (i k h z) \exp (i l \theta)\left\{\left[\frac{i l}{k r} \gamma_{l}(h) J_{l}(\bar{y})\right.\right. \\
& \left.+h\left(1-h^{2} / n^{2}\right)^{1 / 2} \delta_{l}(h) J_{l}^{\prime}(\bar{y})\right] \hat{u}_{r} \\
& -\left[n\left(1-h^{2} / n^{2}\right)^{1 / 2} \gamma_{l}(h) J_{l}^{\prime}(\bar{y})\right. \\
& \left.-\frac{i h l}{n k r} \delta_{l}(h) J_{l}(\bar{y})\right] \hat{u}_{\theta} \\
& \left.-\left[i n\left(1-h^{2} / n^{2}\right) \delta_{l}(h) J_{l}(\bar{y})\right] \hat{u}_{z}\right\},
\end{aligned}
$$

where

$$
\bar{y}=n k r\left(1-h^{2} / n^{2}\right)^{1 / 2}
$$

and the dependence of the interior field on the incidentbeam profile is given by

$$
\begin{aligned}
& \gamma_{l}(h)=c_{l}(h) A_{l}(h)+p_{l}(h) B_{l}(h), \\
& \delta_{l}(h)=-n p_{l}(h) A_{l}(h)+d_{l}(h) B_{l}(h) .
\end{aligned}
$$

For an incident plane wave, again the $h$ integral in Eq. (16) may be evaluated exactly, since $A_{l}(h)$ and $B_{l}(h)$ are $\delta$ functions. But for focused-Gaussian-beam incidence, the $h$ integral cannot be approximated by the stationaryphase method.

\section{PARTIAL-WAVE TRANSMISSION AND REFLECTION FRESNEL COEFFICIENTS}

The derivation of the partial-wave Fresnel coefficients in this section proceeds in analogy to the derivation in Appendix A of Ref. 12 of the analogous quantities for scattering by a sphere. We define the region exterior to the cylinder to be region 2 and the cylinder interior to be region 1. Consider a single $\epsilon$-polarized radially incoming partial wave with unit amplitude and with indices $l$ and $h$, which is incident on the cylinder surface from region 2 . The scalar radiation potential of the partial wave is

$$
\psi_{\text {inc }}^{\epsilon}(r, \theta, z)=H_{l}^{(2)}\left(k r\left(1-h^{2}\right)^{1 / 2}\right) \exp (i k h z) \exp (i l \theta) .
$$

Upon interaction with the cylinder surface, a portion of the partial wave is reflected back into region 2 with either $\epsilon$ or $\mu$ polarization, and the remaining portion is transmitted into region 1 with either $\epsilon$ or $\mu$ polarization. The radially outgoing reflected radiation potentials in region 2 are

$$
\begin{aligned}
& \psi_{\mathrm{ref}}^{\epsilon}(r, \theta, z)=R_{\epsilon \epsilon}^{22} H_{l}^{(1)}\left(k r\left(1-h^{2}\right)^{1 / 2}\right) \exp (i k h z) \exp (i l \theta), \\
& \psi_{\mathrm{ref}}^{\mu}(r, \theta, z)=R_{\epsilon \mu}^{22} H_{l}^{(1)}\left(k r\left(1-h^{2}\right)^{1 / 2}\right) \exp (i k h z) \exp (i l \theta),
\end{aligned}
$$

and the radially incoming transmitted radiation potentials in region 1 are

$$
\begin{aligned}
\psi_{\text {trans }}^{\epsilon}(r, \theta, z)= & T_{\epsilon \epsilon}^{21} H_{l}^{(2)}\left(n k r\left(1-h^{2} / n^{2}\right)^{1 / 2}\right) \\
& \times \exp (i k h z) \exp (i l \theta), \\
\psi_{\text {trans }}^{\mu}(r, \theta, z)= & T_{\epsilon \mu}^{21} H_{l}^{(2)}\left(n k r\left(1-h^{2} / n^{2}\right)^{1 / 2}\right) \\
& \times \exp (i k h z) \exp (i l \theta) .
\end{aligned}
$$

The amplitudes of the reflected and transmitted portions, $R_{\epsilon \epsilon}^{22}, R_{\epsilon \mu}^{22}, T_{\epsilon \epsilon}^{21}$, and $T_{\epsilon \mu}^{21}$, are the partial-wave reflection and transmission Fresnel coefficients, respectively. The leftmost superscript and subscript of the Fresnel coefficients denote the initial region and polarization of the partial wave, and the rightmost superscript and subscript denote the final region and polarization of the reflected or transmitted portion. Since the electric and magnetic fields of the partial wave are derivatives of the scalar radiation potentials, ${ }^{10}$ continuity of $E_{\theta}, E_{z}, B_{\theta}$, and $B_{z}$ at $r=a$ provides four algebraic equations from which the four partial-wave Fresnel coefficients may be determined.

There are 16 partial-wave Fresnel coefficients for the diagonal incidence/cylinder geometry considered here. The remaining 12 are obtained by considering a radially incoming $\mu$-polarized partial wave in region 2 incident on the interface and the initially radially outgoing $\epsilon$-polarized and $\mu$-polarized partial waves in region 1 incident on the interface. Continuity of the transverse field components at the cylinder surface generates additional systems of algebraic equations that may then be solved. The result is

$$
\begin{aligned}
R_{\epsilon \epsilon}^{22} & =\frac{n G_{12} G_{22}-E_{22} F_{12}}{D_{12}}, \\
R_{\mu \mu}^{22} & =\frac{n G_{12} G_{22}-F_{22} E_{12}}{D_{12}}, \\
R_{\epsilon \mu}^{22} & =\frac{i}{D_{12}}\left(E_{12} G_{22}-E_{22} G_{12}\right), \\
R_{\mu \epsilon}^{22} & =\frac{-i n}{D_{12}}\left(F_{12} G_{22}-F_{22} G_{12}\right), \\
R_{\epsilon \epsilon}^{11} & =\frac{n G_{12} G_{11}-E_{11} F_{12}}{D_{12}}, \\
R_{\mu \mu}^{11} & =\frac{n G_{12} G_{11}-F_{11} E_{12}}{D_{12}}, \\
R_{\epsilon \mu}^{11} & =\frac{i n}{D_{12}}\left(E_{12} G_{11}-E_{11} G_{12}\right),
\end{aligned}
$$




$$
\begin{gathered}
R_{\mu \epsilon}^{11}=\frac{-i}{D_{12}}\left(F_{12} G_{11}-F_{11} G_{12}\right), \\
T_{\epsilon \epsilon}^{21}=\frac{-4 i n^{2} x F_{12}}{\pi y^{2} D_{12}}, \quad T_{\mu \mu}^{21}=\frac{-4 i n^{2} x E_{12}}{\pi y^{2} D_{12}}, \\
T_{\epsilon \mu}^{21}=\frac{4 n^{3} x G_{12}}{\pi y^{2} D_{12}}, \quad T_{\mu \epsilon}^{21}=\frac{-n^{2} x G_{12}}{\pi y^{2} D_{12}}, \\
T_{\epsilon \epsilon}^{12}=\frac{-4 i F_{12}}{\pi x D_{12}}, \quad T_{\mu \mu}^{12}=\frac{-4 i E_{12}}{\pi x D_{12}}, \\
T_{\epsilon \mu}^{12}=\frac{4 G_{12}}{\pi x D_{12}}, \quad T_{\mu \epsilon}^{12}=\frac{-4 n G_{12}}{\pi x D_{12}},
\end{gathered}
$$

where

$$
\begin{aligned}
E_{i j} & =\frac{n x}{y} H_{l}^{(i)}(x) H_{l}^{(j) \prime}(y)-n H_{l}^{(i) \prime}(x) H_{l}^{(j)}(y), \\
F_{i j} & =\frac{n^{2} x}{y} H_{l}^{(i)}(x) H_{l}^{(j) \prime}(y)-H_{l}^{(i) \prime}(x) H_{l}^{(j)}(y), \\
G_{i j} & =\frac{h l}{x y^{2}}\left(y^{2}-x^{2}\right) H_{l}^{(i)}(x) H_{l}^{(j)}(y), \\
D_{12} & =E_{12} F_{12}-n{G_{12}}^{2} .
\end{aligned}
$$

These partial-wave Fresnel coefficients are the wave theory analog of the familiar reflection and transmission Fresnel coefficients for the interaction of a diagonally incident plane wave with a flat interface. ${ }^{13}$

\section{DEBYE-SERIES EXPANSION OF THE PARTIAL-WAVE SCATTERING AND INTERIOR AMPLITUDES}

In this section we show that the partial-wave scattering and interior amplitudes of Eqs. (2), (3), and (8)-(11) may be written as an infinite series of combinations of the partial-wave reflection and transmission Fresnel coefficients of Eqs. (22). When a partial-wave component of the incident beam is transmitted into the cylinder, a good analogy of what happens thereafter until the partial wave is eventually transmitted back out is provided by the trajectory of a ball in a pinball machine. ${ }^{14}$ Just as the ball can bounce off the bumpers any number of times and in any order before it completes its trajectory, so also the partial wave may internally reflect off the cylinder surface any number of times before it exits the cylinder. Further, each reflection may be either polarization preserving (i.e., $\epsilon \epsilon$ or $\mu \mu$ ) or cross polarized (i.e., $\epsilon \mu$ or $\mu \epsilon$ ). The total wave theory amplitude is then the sum of the amplitudes for all the possibilities that can occur in the scattering. ${ }^{14}$ Motivated by this physically based point of view, we first consider the partial-wave summed interior reflection amplitude in each of the four polarization channels $\mathscr{R}_{\epsilon \epsilon}^{11}, \mathscr{R}_{\epsilon \mu}^{11}, \mathscr{R}_{\mu \epsilon}^{11}$, and $\mathscr{R}_{\mu \mu}^{11}$, where

$$
\begin{aligned}
\mathscr{R}_{i j}^{11}= & R_{i j}^{11}+\sum_{k} R_{i k}^{11} R_{k j}^{11}+\sum_{k} \sum_{l} R_{i k}^{11} R_{k l}^{11} R_{l j}^{11} \\
& +\sum_{k} \sum_{l} \sum_{m} R_{i k}^{11} R_{k l}^{11} R_{l m}^{11} R_{m j}^{11}+\ldots
\end{aligned}
$$

In Eq. (25) we have $i=\epsilon$ or $\mu, j=\epsilon$ or $\mu$, and the sums over $k, l, m$, etc., are sums over the $\epsilon$ and $\mu$ states. A pictorial representation of $\mathscr{R}_{i j}^{11}$ is given in Fig. 1 .

For each of the four polarization channels, the infinite series in Eq. (25) may be evaluated in closed form by using the following argument. Consider first $\mathscr{R}_{\epsilon \epsilon}^{11}$. Each term in Eq. (25) contains a certain number $(r-s \geqslant 1)$ of $R_{\epsilon \epsilon}^{11}$ factors and a certain number $(s \geqslant 0)$ of $R_{\epsilon \mu}^{11} R_{\mu \epsilon}^{11}$ factors. The cross-polarized factors always occur as a pair because the polarization must return to the $\epsilon$ state by the end of the interaction. The $R_{\epsilon \mu}^{11} R_{\mu \epsilon}^{11}$ factors may occur at any step along the way, and any number of factors (st $\geqslant 0$ ) of $R_{\mu \mu}^{11}$ may occur between $R_{\epsilon \mu}^{11}$ and $R_{\mu \epsilon}^{11}$. Upon enumerating all the possibilities for successive internal reflections, we obtain

$$
\begin{aligned}
\mathscr{R}_{\epsilon \epsilon}^{11}= & \sum_{r=1}^{\infty} \sum_{s=0}^{r} \sum_{t=0}^{\infty} \frac{r !}{s !(r-s) !} \\
& \times\left(R_{\epsilon \epsilon}^{11}\right)^{r-s}\left(R_{\epsilon \mu}^{11}\right)^{s}\left(R_{\mu \mu}^{11}\right)^{s t}\left(R_{\mu \epsilon}^{11}\right)^{s} \\
= & \frac{R_{\epsilon \epsilon}^{11}\left(1-R_{\mu \mu}^{11}\right)+R_{\epsilon \mu}^{11} R_{\mu \epsilon}^{11}}{\left(1-R_{\epsilon \epsilon}^{11}\right)\left(1-R_{\mu \mu}^{11}\right)-R_{\epsilon \mu}^{11} R_{\mu \epsilon}^{11}} .
\end{aligned}
$$

The closed-form expression for $\mathscr{R}_{\mu \mu}^{11}$ is obtained from Eq. (26) by the replacement $\epsilon \leftrightarrow \mu$. For $\mathscr{R}_{\epsilon \mu}^{11}$ the situation is similar, except that there is an extra factor of $R_{\epsilon \mu}^{11}$ to produce the cross polarization and there is no stand-alone factor of $R_{\epsilon \epsilon}^{11}$ as in the first line of Eq. (26). Upon enumerating all the possibilities for successive internal reflections, we obtain

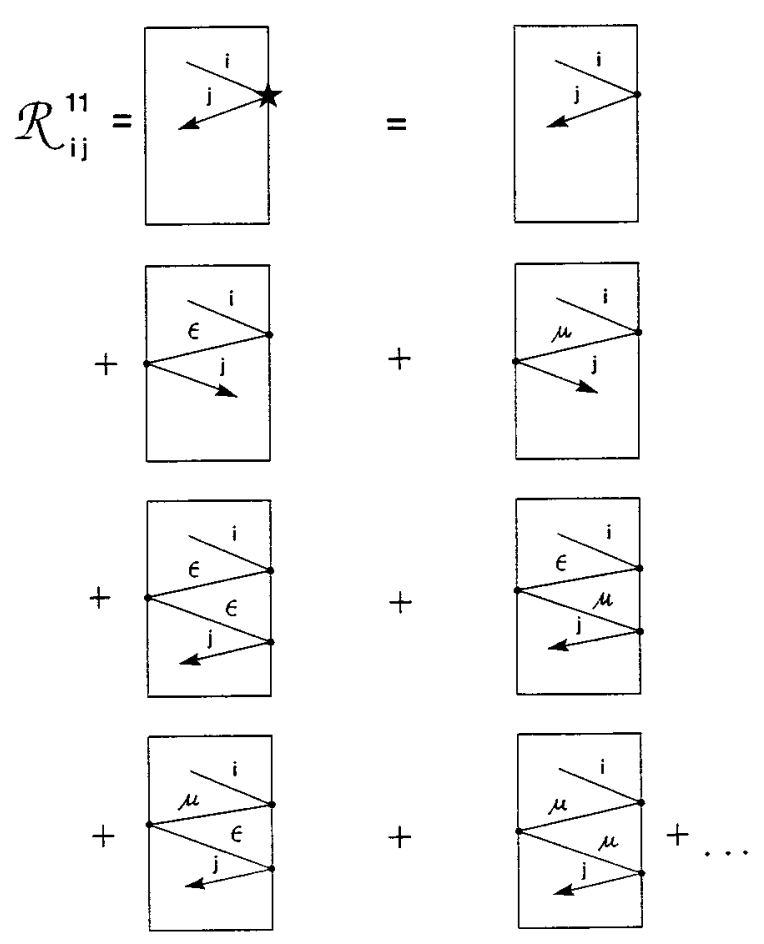

Fig. 1. Pictorial representation of the partial-wave summed interior reflection amplitude $\mathscr{K}_{i j}^{11}$ for initial polarization state $i$ and final polarization state $j$. Each dot is an $R^{11}$ factor. 


$$
\mathscr{R}_{\epsilon \mu}^{11}=\frac{R_{\epsilon \mu}^{11}}{\left(1-R_{\epsilon \epsilon}^{11}\right)\left(1-R_{\mu \mu}^{11}\right)-R_{\epsilon \mu}^{11} R_{\mu \epsilon}^{11}} .
$$

The closed-form expression for $\mathscr{R}_{\mu \epsilon}^{11}$ is obtained from Eq. (27) by the replacement $\epsilon \leftrightarrow \mu$.

Upon substituting Eqs. (22) into Eqs. (26) and (27) and after much algebraic simplification, we may write the partial-wave interior amplitudes of Eqs. (3), (9), and (11) in Debye-series form as

$$
\begin{aligned}
c_{l}(h)= & \frac{1}{n}\left(T_{\epsilon \epsilon}^{21}+\sum_{k} T_{\epsilon k}^{21} \mathscr{R}_{k \epsilon}^{11}\right), \\
d_{l}(h)= & \frac{1}{n}\left(T_{\mu \mu}^{21}+\sum_{k} T_{\mu k}^{21} \mathscr{R}_{k \mu}^{11}\right), \\
p_{l}(h)= & \frac{1}{n}\left(T_{\mu \epsilon}^{21}+\sum_{k} T_{\mu k}^{21} \mathscr{R}_{k \epsilon}^{11}\right) \\
= & \frac{-1}{n^{2}}\left(T_{\epsilon \mu}^{21}+\sum_{k} T_{\epsilon k}^{21} \mathscr{R}_{k \mu}^{11}\right),
\end{aligned}
$$

where the $k$ sum is over the $\epsilon$ and $\mu$ states. A pictorial representation of Eqs. (28), (29), and (30) is given in Figs. 2(a), 2(b), and 2(c), respectively. The interior-amplitude Debye series makes a good deal of physical sense. A partial wave inside the cylinder either has been directly transmitted or has been transmitted and then has participated in any number of internal reflections within the cylinder. At the transmission and at each internal reflection, the interaction with the cylinder surface has both polarization-preserving and cross-polarized components.
The partial-wave interior amplitudes of Eqs. (28)-(30) provide a starting point for the Debye-series expansion of the partial-wave scattering amplitudes of Eqs. (2), (8), and (10), since postmultiplication of the interior amplitudes by a $T_{i j}^{12}$ factor transmits the partial wave from the cylinder interior back into the exterior region. Again after much algebra we obtain

$$
\begin{aligned}
a_{l}(h)= & \frac{1}{2}-\frac{1}{2}\left(R_{\epsilon \epsilon}^{22}+\sum_{k} T_{\epsilon k}^{21} T_{k \epsilon}^{12}\right. \\
& \left.+\sum_{k} \sum_{l} T_{\epsilon k}^{21} \mathscr{R}_{k l}^{11} T_{l \epsilon}^{12}\right), \\
b_{l}(h)= & \frac{1}{2}-\frac{1}{2}\left(R_{\mu \mu}^{22}+\sum_{k} T_{\mu k}^{21} T_{k \mu}^{12}\right. \\
& \left.+\sum_{k} \sum_{l} T_{\mu k}^{21} \mathscr{B}_{k l}^{11} T_{l \mu}^{12}\right), \\
& \frac{-1}{2}\left(R_{\mu \epsilon}^{22}+\sum_{k} T_{\mu k}^{21} T_{k \epsilon}^{12}+\sum_{k} \sum_{l} T_{\mu k}^{21} \mathscr{B}_{k l}^{11} T_{l \epsilon}^{12}\right) \\
q_{l}(h)= & \frac{1}{2}\left(R_{\epsilon \mu}^{22}+\sum_{k} T_{\epsilon k}^{21} T_{k \mu}^{12}+\sum_{k} \sum_{l} T_{\epsilon k}^{21} \mathscr{B}_{k l}^{11} T_{l \mu}^{12}\right),
\end{aligned}
$$

where the $k$ and $l$ sums are over the $\epsilon$ and $\mu$ states. A pictorial representation of Eqs. (31), (32), and (33) is given in Figs. 3(a), 3(b), and 3(c), respectively. The leading factor of $1 / 2$ in Eqs. (31) and (32) represents diffraction. ${ }^{15}$ The polarization-preserving amplitudes $a_{l}(h)$ and $b_{l}(h)$
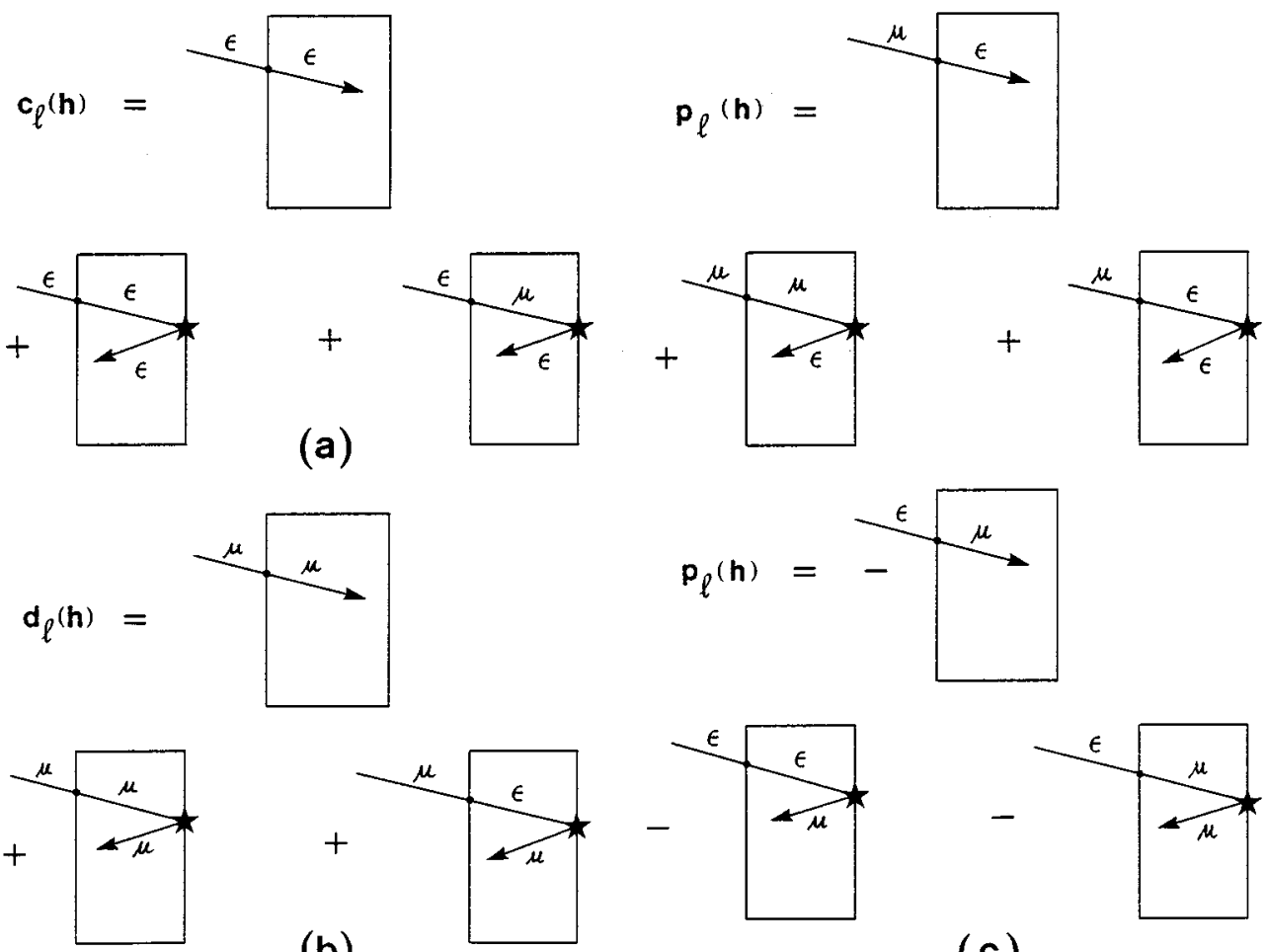

(b)

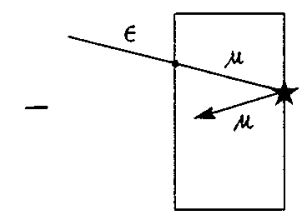

(c)

Fig. 2. Pictorial representation of the partial-wave interior amplitudes (a) $c_{l}(h)$ for the $\epsilon \rightarrow \epsilon$ polarization channel, (b) $d_{l}(h)$ for the $\mu \rightarrow \mu$ channel, and (c) $p_{l}(h)$ for the $\mu \rightarrow \epsilon$ and $\epsilon \rightarrow \mu$ channels. Each dot is a $T^{21}$ factor, and each star is an $\mathscr{B}^{11}$ factor. 


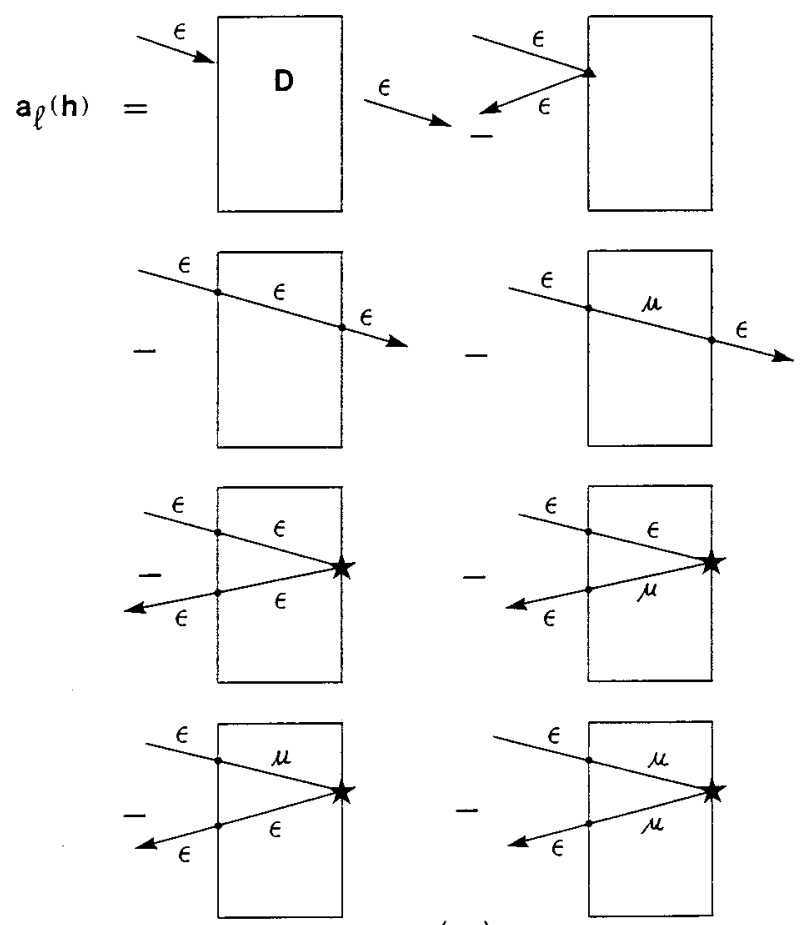

(a)
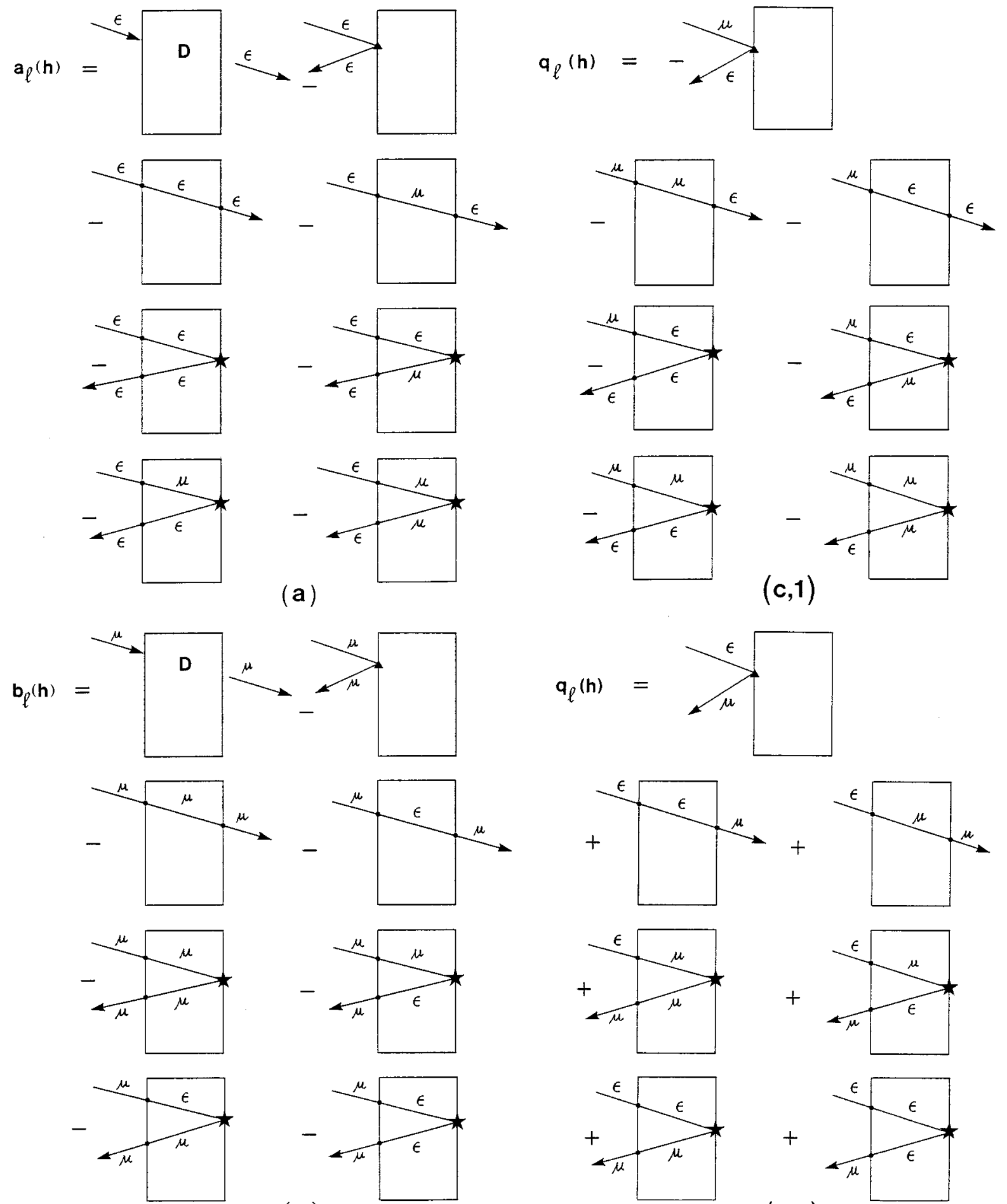

(b)
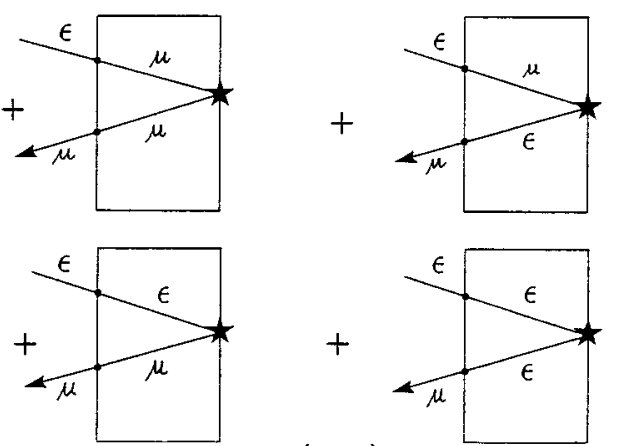

Fig. 3. Pictorial representation of the partial-wave scattering amplitudes (a) $a_{l}(h)$ for $\epsilon \rightarrow \epsilon$ scattering, (b) $b_{l}(h)$ for $\mu \rightarrow \mu$ scattering, and (c) $q_{l}(h)$ for the $\mu \rightarrow \epsilon$ and $\epsilon \rightarrow \mu$ scatterings. Each dot is a $T^{21}$ or $T^{12}$ factor, each star is an $\mathscr{B}^{11}$ factor, each triangle is an $R^{22}$ factor, and $D$ represents diffraction.

either can keep the partial wave in the same polarization state throughout or can switch the polarization state of the partial wave at any one of the interactions with the surface, so long as the polarization switches back before the partial wave leaves the cylinder. The cross-polarized amplitude $q_{l}(h)$ can switch the polarization state of the partial wave at any one of the interactions with the surface. This polarization switching explains why the partial-wave scattering amplitudes $a_{l}(h), b_{l}(h)$, and $q_{l}(h)$ each exhibit both $\mathrm{TE}_{l, m}$ and $\mathrm{TM}_{l, m}$ morphologydependent resonances at diagonal incidence, ${ }^{11}$ whereas at normal incidence, where all the interactions with the cyl- 
inder surface are polarization preserving, $a_{l}(0)$ exhibits only $\mathrm{TE}_{l, m}$ resonances and $b_{l}(0)$ exhibits only $\mathrm{TM}_{l, m}$ resonances.

The Debye-series decomposition of the partial-wave scattering and interior amplitudes has a long history. It has been derived for electromagnetic scattering by a cylinder at normal incidence, ${ }^{16}$ for scattering by a homogeneous sphere, $8,17,18$ and for scattering by a coated sphere. ${ }^{19-21}$ Since scattering for those three geometries is polarization preserving, the Debye series simplifies substantially. For scattering by a cylinder at normal incidence or by a sphere, there are eight partial-wave Fresnel coefficients that couple together in two groups of 4 (i.e., the TE coefficients couple together, and the TM coefficients couple together) to form the partial-wave scattering and interior amplitudes. For scattering by a coated sphere, there are 16 partial-wave Fresnel coefficients that couple together in two groups of 8 to form the partial-wave scattering and interior amplitudes. For the diagonal incidence/cylinder scattering considered here, again there are 16 partial-wave Fresnel coefficients in Eqs. (22). But, on account of cross-polarized scattering, all 16 couple together in Eqs. (28)-(33) to form the partial-wave scattering and interior amplitudes. Since Eqs. (28)-(33) are valid for arbitrary $h$, our Debye-series decomposition is valid for an incident beam of arbitrary profile.

The terms of the Debye series in classical electromagnetic scattering are analogous to Feynman diagrams for scattering in quantum electrodynamics. ${ }^{22}$ In each case the total scattering amplitude is decomposed into the infinite series of all the different possible physical processes that can occur in the situation at hand. The Debye series, however, has not enjoyed the widespread use that Feynman diagrams have. This is because in quantum electrodynamics, the total scattering amplitude cannot be evaluated in closed form. Thus the calculation of cross sections must be performed by evaluating and adding together the amplitudes of dominant physical processes that occur in the scattering situation. In classical electromagnetic scattering, on the other hand, the total scattering amplitude can be calculated exactly for a target particle having a high degree of symmetry. The Debye series then plays less of a calculational role, and its main use is to provide an intuitive understanding of the different physical processes that contribute to scattering.

The Debye series may also be interpreted as being the solution to a particular type of multiple-scattering problem, since the scattering amplitudes $a_{l}(h), b_{l}(h)$, and $q_{l}(h)$ result from the infinite series of interactions of partial waves with the cylinder surface. Since both polarization-preserving and cross-polarized scattering occur for the diagonal incidence/cylinder geometry, the Debye series of Eqs. (31)-(33) serves as an exactly soluble prototype example of a wave that may change its internal state (i.e., from $\epsilon$ to $\mu$ or from $\mu$ to $\epsilon$ ) at each interaction during multiple scattering. Such state-changing interactions occur frequently and are of great interest in quantum-mechanical scattering. The Debye series is also the series expansion of the interaction $S$ matrix for scattering as considered in Ref. 11.

Last, the Debye-series results of Eqs. (28)-(33) simplify substantially for two special cases. For scattering by a normally incident plane wave, we have $h=0$, and Eqs. (26) and (27) reduce to

$$
\begin{gathered}
\mathscr{R}_{\epsilon \epsilon}^{11}=\frac{R_{\epsilon \epsilon}^{11}}{1-R_{\epsilon \epsilon}^{11}}, \quad \mathscr{R}_{\mu \mu}^{11}=\frac{R_{\mu \mu}^{11}}{1-R_{\mu \mu}^{11}}, \\
\mathscr{R}_{\mu \epsilon}^{11}=\mathscr{R}_{\epsilon \mu}^{11}=0 .
\end{gathered}
$$

These expressions lead to the normal-incidence Debye series

$$
\begin{aligned}
& c_{l}(0)=\frac{1}{n} \sum_{p=1}^{\infty} T_{\epsilon \epsilon}^{21}\left(R_{\epsilon \epsilon}^{11}\right)^{p-1}, \\
& d_{l}(0)=\frac{1}{n} \sum_{p=1}^{\infty} T_{\mu \mu}^{21}\left(R_{\mu \mu}^{11}\right)^{p-1}, \\
& p_{l}(0)=0 \\
& a_{l}(0)=\frac{1}{2}-\frac{1}{2} \sum_{p=1}^{\infty} T_{\epsilon \epsilon}^{21}\left(R_{\epsilon \epsilon}^{11}\right)^{p-1} T_{\epsilon \epsilon}^{12}, \\
& b_{l}(0)=\frac{1}{2}-\frac{1}{2} \sum_{p=1}^{\infty} T_{\mu \mu}^{21}\left(R_{\mu \mu}^{11}\right)^{p-1} T_{\mu \mu}^{12}, \\
& q_{l}(0)=0,
\end{aligned}
$$

which was first derived by Debye ${ }^{16}$ and for whom the series is named.

For scattering of a diagonally incident beam of arbitrary profile by a perfectly conducting cylinder, we have $n \rightarrow \infty$ and

$$
\begin{aligned}
& a_{l}(h)=\frac{J_{l}^{\prime}(x)}{H_{l}^{(1)^{\prime}(x)}}=\frac{1}{2}\left(1-R_{\epsilon \epsilon}^{22}\right), \\
& b_{l}(h)=\frac{J_{l}(x)}{H_{l}^{(1)}(x)}=\frac{1}{2}\left(1-R_{\mu \mu}^{22}\right), \\
& q_{l}(h)=0
\end{aligned}
$$

where

$$
R_{\epsilon \epsilon}^{22}=-H_{l}^{(2)^{\prime}}(x) / H_{l}^{(1)^{\prime}}(x), \quad R_{\mu \mu}^{22}=-H_{l}^{(2)}(x) / H_{l}^{(1)}(x) .
$$

No cross-polarized scattering occurs, and the only physical processes contributing to polarization-preserving scattering are diffraction and reflection.

\section{FIRST-ORDER RAINBOW FOR SCATTERING OF A DIAGONALLY INCIDENT PLANE WAVE BY A CYLINDER}

In Ref. 23 it was demonstrated in the context of ray theory that there is a qualitative similarity between varying the tilt angle $\xi$ of the incident plane wave and varying the refractive index $n$ at normal incidence. As $\xi$ increases (or as $n$ increases at normal incidence), the scattering angle of the first-order rainbow also increases. The scattering angle reaches $180^{\circ}$ when

$$
\sin \xi_{e}=\left(\frac{4-n^{2}}{3}\right)^{1 / 2} \text {. }
$$


A further increase in $\xi$ past this value extinguishes the rainbow. Thus we term $\xi_{e}$ the rainbow extinction tilt angle. Also at $\xi=\xi_{e}$, the $p=1$ interior cusp caustic has its cusp point focal line touching the surface of the cylinder. In this section we examine this rainbow extinction transition by using the Debye-series partial-wave scattering amplitudes. For definiteness we employ the refractive index $n=1.484$ corresponding to the glass rod of Ref. 23, which gives $\xi_{e}=50.72^{\circ}$. We also employ the cylinder size parameter $2 \pi a / \lambda=1000.0$, which is substantially less than that of the glass rod of Ref. 23 but which satisfies the short-wavelength-limit criterion nonetheless.

As mentioned in Section 2, the expression for the scattered intensity simplifies considerably for diagonal planewave incidence. If the incident beam is an $\epsilon$-polarized plane wave, its beam-shape coefficients are

$$
A_{l}(h)=\delta(h+\sin \xi), \quad B_{l}(h)=0 ;
$$

and if the incident plane wave is $\mu$ polarized, its beamshape coefficients are

$$
A_{l}(h)=0, \quad B_{l}(h)=\delta(h+\sin \xi) .
$$

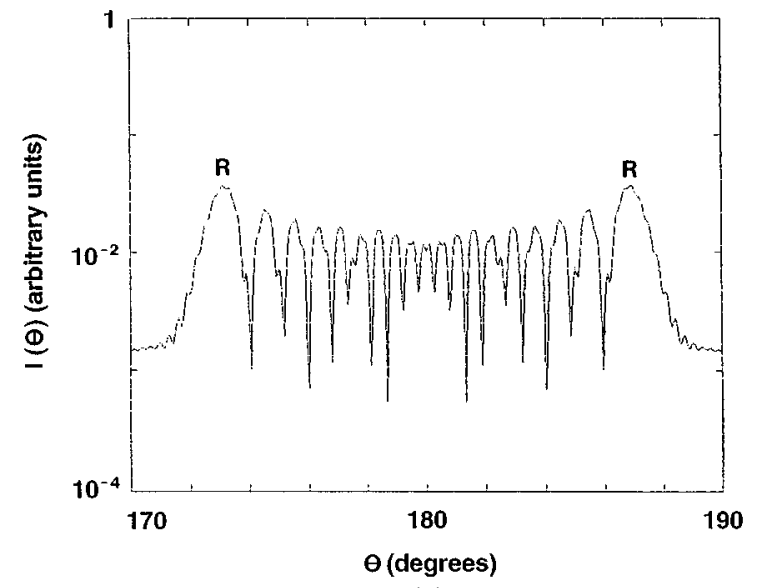

(a)

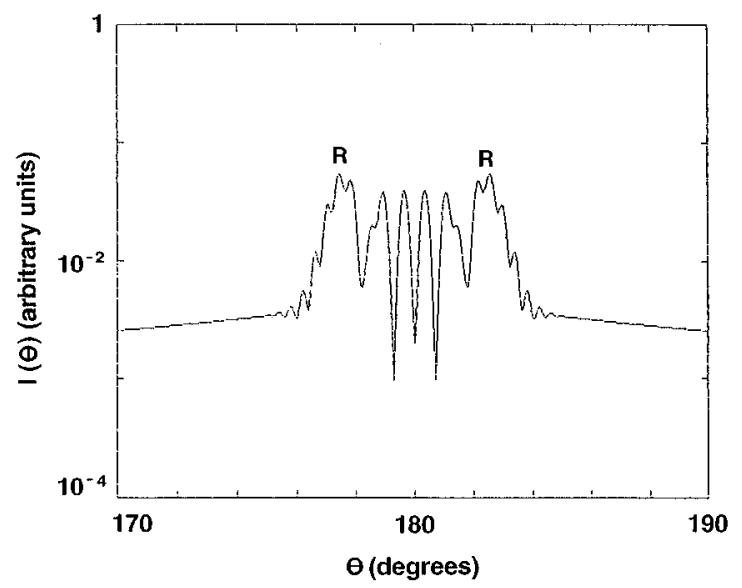

(b)
For an unpolarized incident plane wave, substitution of Eqs. (40) and (41) into Eqs. (12)-(14) gives the far-zone scattered intensity

$$
\begin{aligned}
\lim _{r \rightarrow \infty} I_{\text {scatt }}(r, \theta, z)= & \left(\frac{E_{0}^{2}}{2 \mu_{0} c}\right)\left(\frac{1}{\pi k r \cos \xi}\right)\left[\left|S_{\epsilon}(\theta)\right|^{2}\right. \\
& \left.+\left|S_{\mu}(\theta)\right|^{2}+2\left|S_{q}(\theta)\right|^{2}\right]
\end{aligned}
$$

where

$$
\begin{aligned}
& S_{\epsilon}(\theta)=a_{0}+2 \sum_{l=1}^{\infty} a_{l} \cos (l \theta), \\
& S_{\mu}(\theta)=b_{0}+2 \sum_{l=1}^{\infty} b_{l} \cos (l \theta), \\
& S_{q}(\theta)=2 i \sum_{l=1}^{\infty} q_{l} \sin (l \theta)
\end{aligned}
$$

and $\mu_{0}$ is the permeability of free space. The partialwave scattering amplitudes $a_{l}, b_{l}$, and $q_{l}$ are given

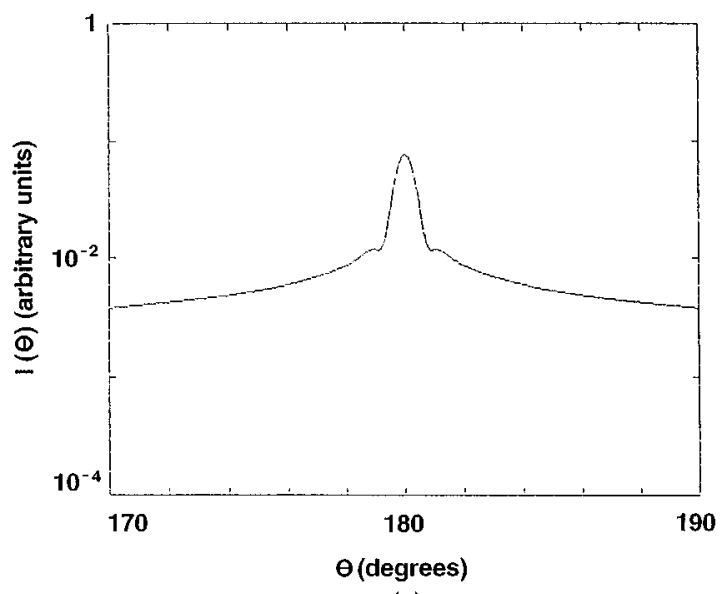

(c)

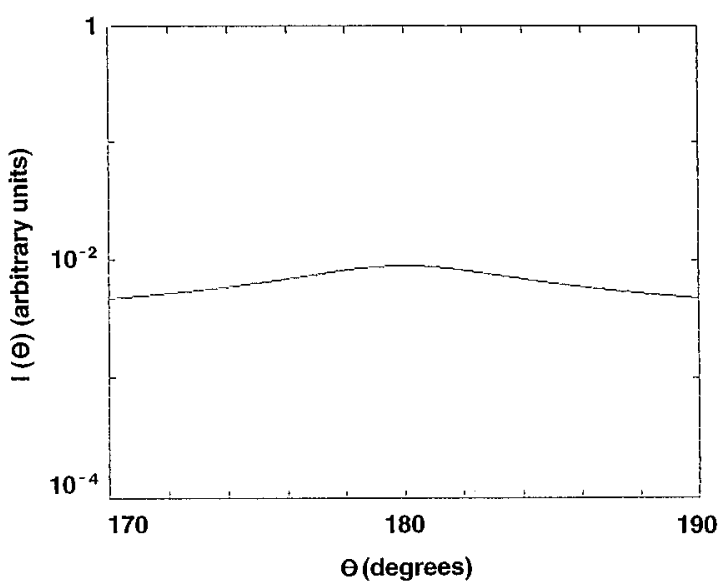

(d)

Fig. 4. One-internal-reflection portion of the scattered intensity as a function of the scattering angle $\theta$ for $n=1.484,2 \pi a / \lambda$ $=1000.0$, and (a) $\xi=39.0^{\circ}$, (b) $\xi=45.0^{\circ}$, (c) $\xi_{e}=50.72^{\circ}$, and (d) $\xi=56.0^{\circ}$. The points labeled $R$ in (a) and (b) are the two branches of the first-order rainbow. 


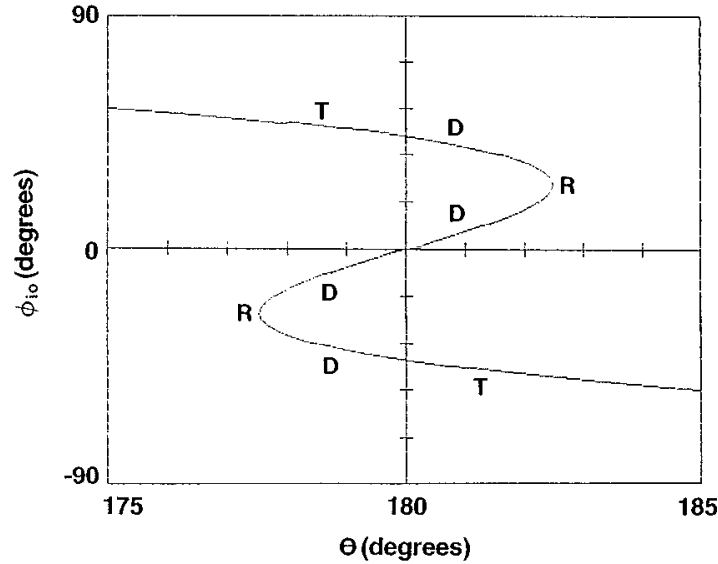

(a)

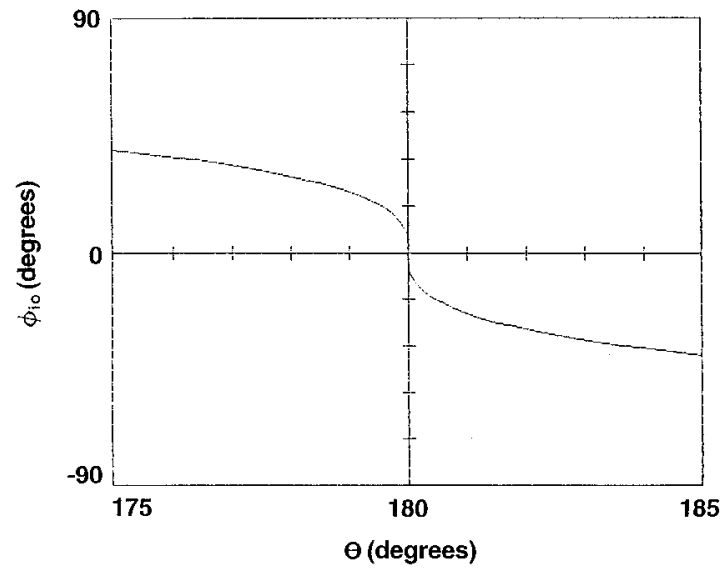

(b)

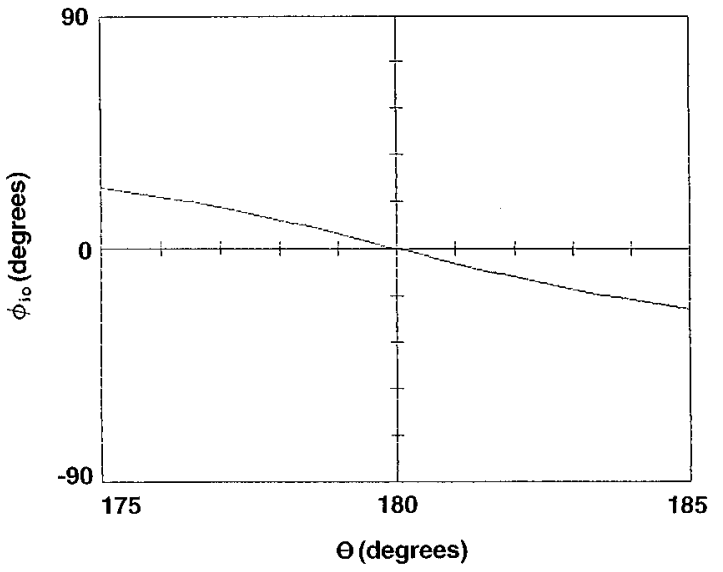

(c)

Fig. 5. Relation between the scattering angle $\theta$ and the impact parameter of a diagonally incident ray parameterized by the angle $\phi_{i 0}$ for $n=1.484$ and (a) $\xi=45.0^{\circ}$, (b) $\xi_{e}=50.72^{\circ}$, and (c) $\xi=56.0^{\circ}$. The points labeled $R$ in (a) are the two branches of the first-order rainbow, the points labeled $D$ are the two dominant rays in the supernumerary region, and the points labeled $T$ are the third ray.

by Eqs. (2), (8), and (10) with $h=-\sin \xi$. The oneinternal-reflection portion of the partial-wave scattering amplitudes is

$$
\begin{aligned}
a_{l} \approx & -\frac{1}{2}\left(T_{\epsilon \epsilon}^{21} R_{\epsilon \epsilon}^{11} T_{\epsilon \epsilon}^{12}+T_{\epsilon \mu}^{21} R_{\mu \epsilon}^{11} T_{\epsilon \epsilon}^{12}+T_{\epsilon \epsilon}^{21} R_{\epsilon \mu}^{11} T_{\mu \epsilon}^{12}\right. \\
& \left.+T_{\epsilon \mu}^{21} R_{\mu \mu}^{11} T_{\mu \epsilon}^{12}\right), \\
b_{l} \approx & -\frac{1}{2}\left(T_{\mu \mu}^{21} R_{\mu \mu}^{11} T_{\mu \mu}^{12}+T_{\mu \epsilon}^{21} R_{\epsilon \mu}^{11} T_{\mu \mu}^{12}+T_{\mu \mu}^{21} R_{\mu \epsilon}^{11} T_{\epsilon \mu}^{12}\right. \\
& \left.+T_{\mu \epsilon}^{21} R_{\epsilon \epsilon}^{11} T_{\epsilon \mu}^{12}\right), \\
& \\
q_{l} \approx & -\frac{1}{2}\left(T_{\mu \mu}^{21} R_{\mu \mu}^{11} T_{\mu \epsilon}^{12}+T_{\mu \epsilon}^{21} R_{\epsilon \mu}^{11} T_{\mu \epsilon}^{12}+T_{\mu \mu}^{21} R_{\mu \epsilon}^{11} T_{\epsilon \epsilon}^{12}\right. \\
& \left.+T_{\mu \epsilon}^{21} R_{\epsilon \epsilon}^{11} T_{\epsilon \epsilon}^{12}\right) .
\end{aligned}
$$

The scattered intensity of Eq. (42) for an unpolarized incident beam and with the use of the one-internalreflection partial-wave scattering amplitudes of relations (44)-(46) was computed for $n=1.484,2 \pi a / \lambda=1000.0$, and $\xi=39.0^{\circ}, 45.0^{\circ}, 50.72^{\circ}$, and $56.0^{\circ}$. The method of computation was a generalization to diagonal incidence of the method of Appendix $\mathrm{C}$ of Ref. 24. The results are given in Figs. 4(a), 4(b), 4(c), and 4(d), respectively. These intensity graphs are easily interpreted by referring to Fig. 5 , in which we plot the scattering angle $\theta$ in the horizontal plane as a function of the impact parameter of a light ray incident on the cylinder and making one internal reflection before exiting. Specifically, the angle $\phi_{i 0}$ employed in Fig. 5 is the angle between the projection of the incident ray in the $x-y$ plane and the normal to the cylinder at the point of incidence. The angle $\phi_{i 0}$ varies from $-90^{\circ}$ to $+90^{\circ}$ as the impact parameter of the ray varies from one edge of the cylinder to the other. Figures $5(\mathrm{a}), 5(\mathrm{~b})$, and $5(\mathrm{c})$ correspond to $\xi=45.0^{\circ}, 50.72^{\circ}$, and $56.0^{\circ}$, respectively.

At a tilt angle of $45.0^{\circ}$, the points labeled $R$ in Fig. 5(a) are the extrema of the scattering angle as a function of the ray impact parameter and correspond to the branches of the first-order rainbow in Fig. 4(b) at $\theta=177.5^{\circ}$ and $182.5^{\circ}$. Three rays contribute to the scattered field at each angle between the two rainbow branches, and one ray contributes at each angle to either side of the rainbow region. This behavior is evident in Figs. 4(a) and 4(b). Between the two branches of the first-order rainbow, again labeled $R$, is the supernumerary pattern of the two dominant rays [labeled D in Fig. 5(a)], which possesses some irregularities produced by interference with the third ray [labeled $\mathrm{T}$ in Fig. 5(a)]. The one-internalreflection intensity is nearly constant to either side of the rainbow region, since the intensity in that region is due to the third ray alone.

Figures 4(c) and 5(b) correspond to the rainbow extinction tilt angle $\xi_{e}=50.72^{\circ}$. The pair of scattering angle extrema have coalesced into a single critical point at $\theta$ $=180^{\circ}$. Thus a range of paraxial rays constructively interferes to form the single rainbow peak at $\theta=180^{\circ}$ in Fig. 4(c). As is seen in Fig. 4(d), this single rainbow peak quickly dissipates as the tilt angle is increased past $\xi_{e}$, since the angular region beyond the rainbow peak is described by a complex ray whose intensity falls off faster than exponentially ${ }^{25}$ as a function of $\theta$ and the third ray of Fig. 5(c). As a result, the one-internal-reflection intensity of Fig. 4(d) is again nearly constant. 


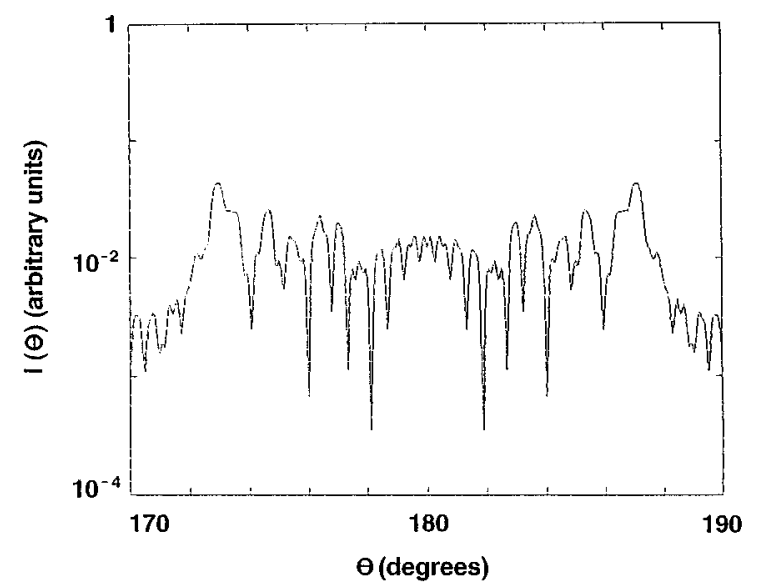

(a)

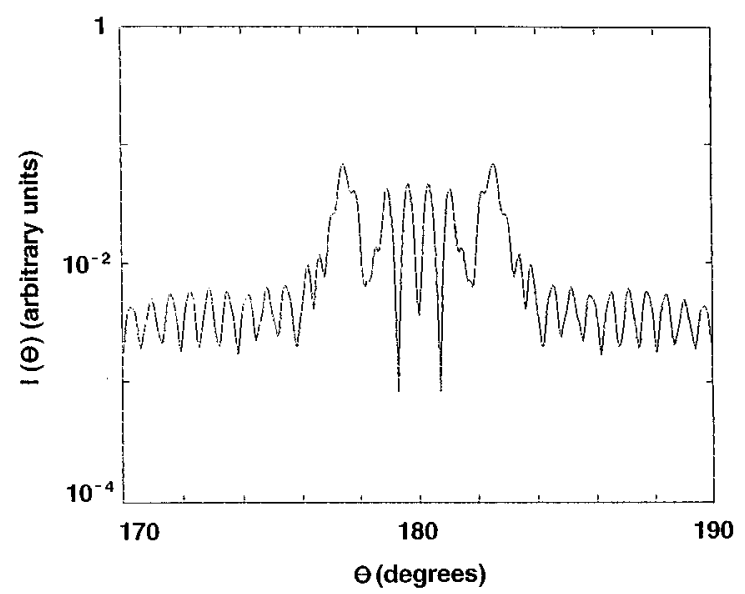

(b)

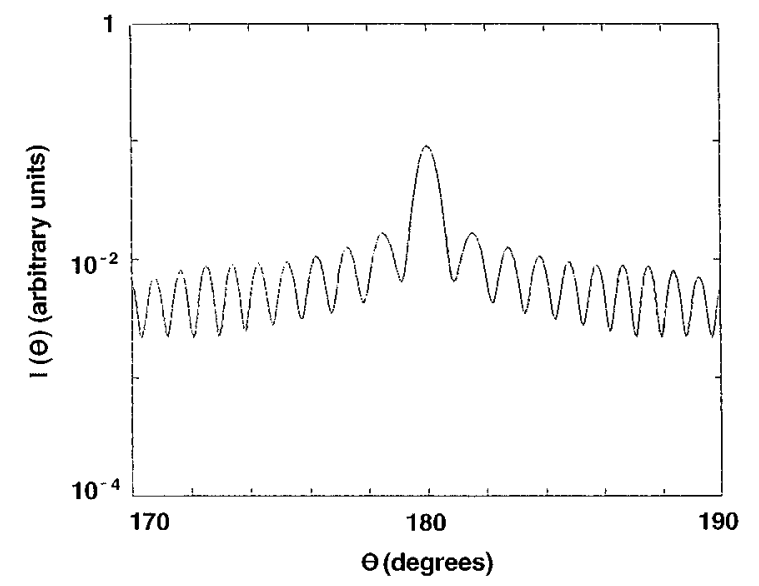

(c)

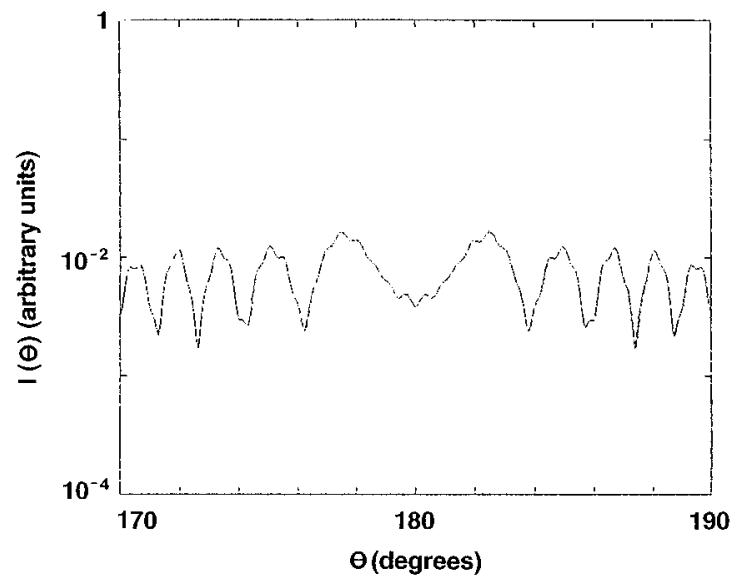

(d)

Fig. 6. Scattered intensity as a function of the scattering angle $\theta$ for $n=1.484,2 \pi a / \lambda=1000.0$, and (a) $\xi=39.0^{\circ}$, (b) $\xi=45.0^{\circ}$, (c) $\xi_{e}=50.72^{\circ}$, and (d) $\xi=56.0^{\circ}$. These graphs are to be compared with the one-internal-reflection portion of the intensity of Figs. $4(\mathrm{a})$, $4(\mathrm{~b}), 4(\mathrm{c})$, and $4(\mathrm{~d})$, respectively.

The scattered intensity associated with the complete partial-wave scattering amplitudes of Eqs. (2), (8), and (10) is shown in Figs. 6(a), 6(b), 6(c), and 6(d) for $n=1.484,2 \pi a / \lambda=1000.0, \quad$ and $\xi=39.0^{\circ}, \quad 45.0^{\circ}$, $50.72^{\circ}$, and $56.0^{\circ}$, respectively. These intensity graphs are to be compared with Figs. 4(a), 4(b), 4(c), and 4(d), respectively. The one-internal-reflection electric field interferes with the $p=0$ reflected electric field, producing both additional distortion to the supernumerary pattern between the two rainbow branches and an oscillatory behavior to either side of the rainbow region. In Fig. 6(d) the high-frequency oscillations in the intensity are produced by interference with the $p=4$ rays that also exit the cylinder in the backscattered direction.

\section{EXPERIMENTAL OBSERVATION OF THE FIRST-ORDER RAINBOW}

The apparatus was described in Ref. 23 and is illustrated in Fig. 7. Briefly, the $\lambda=0.6328 \mu \mathrm{m}$ unpolarized beam

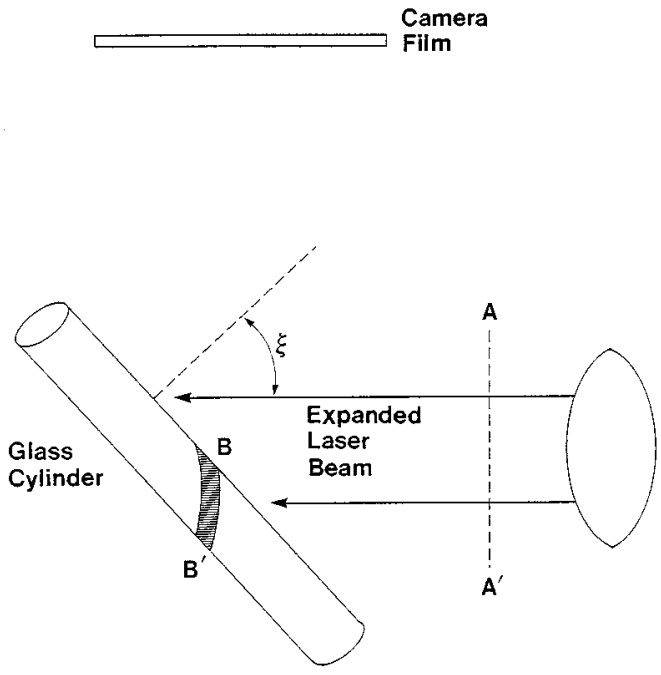

Fig. 7. A laser beam, expanded by a series of lenses, is incident on a 7.6-mm-radius glass rod. Light scattered near $180^{\circ}$ is recorded on unexposed camera film $72 \mathrm{~cm}$ above the rod. A black card with a narrow slit cut into it is placed at $\mathrm{AA}^{\prime}$ so as to illuminate a narrow band $\mathrm{BB}^{\prime}$ on the rod. 


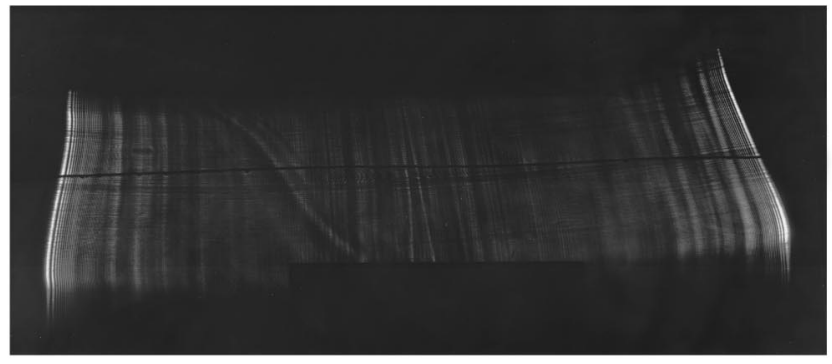

(a)

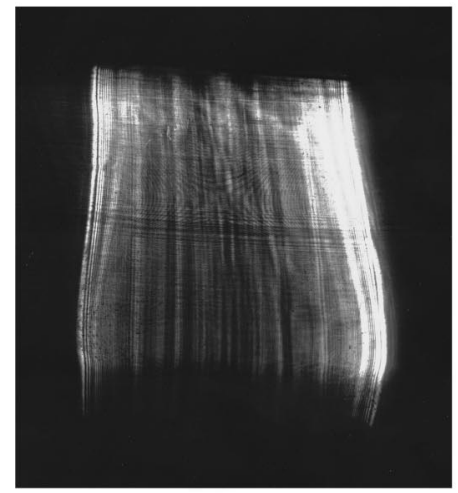

(b)

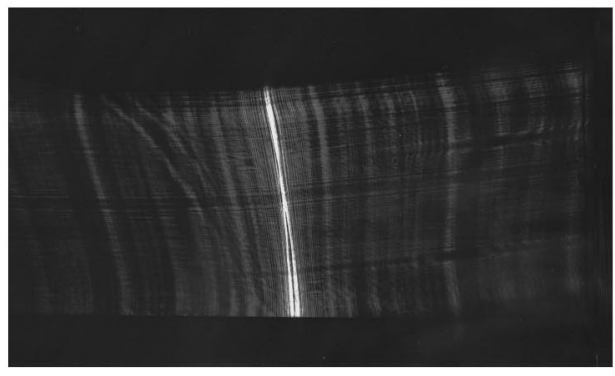

(c)

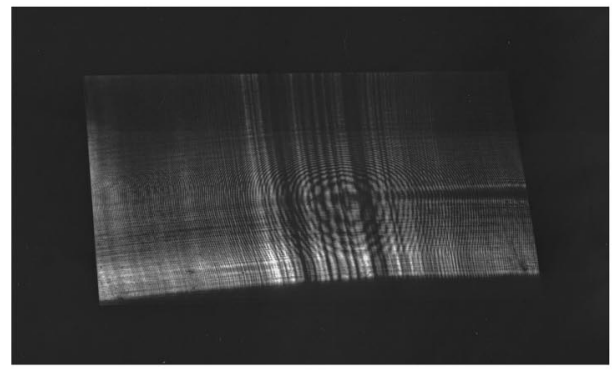

(d)

Fig. 8. Photographs of the first-order rainbow region for (a) $\xi$ $=33^{\circ}$, (b) $\xi=40^{\circ}$, (c) $\xi_{e}=45^{\circ}$, and (d) $\xi=50^{\circ}$.

of a 15-mW HeNe laser was expanded by a series of lenses to a diameter of $5.0 \mathrm{~cm}$. The expanded beam was incident on a glass rod of nominal radius $a=7.6 \mathrm{~mm}$ and refractive index $n=1.484$, which was mounted on a rotation stage so as to vary the tilt angle of the rod easily with respect to the beam. Light scattered near $180^{\circ}$ was re-

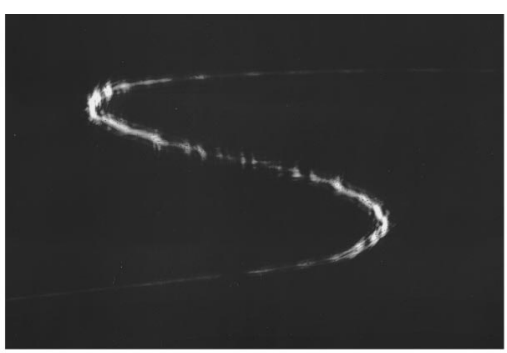

(a)

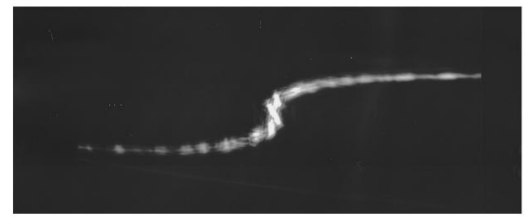

(b)

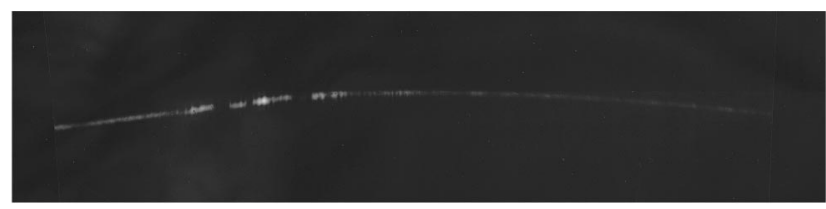

(c)

Fig. 9. Photographs of the first-order rainbow region for narrow-band illumination of the glass rod for (a) $\xi=40^{\circ}$, (b) $\xi_{e}$ $=45^{\circ}$, and (c) $\xi=50^{\circ}$. These photographs are to be compared with the ray theory predictions of Figs. 5(a), 5(b), and 5(c), respectively.

corded on a strip of unexposed photographic film a distance of $72 \mathrm{~cm}$ above the glass rod.

Photographs of the first-order rainbow region are shown in Figs. 8(a), 8(b), 8(c), and 8(d) for $\xi=33^{\circ}, 40^{\circ}$, $45^{\circ}$, and $50^{\circ}$, respectively. It was found that the rainbow extinction tilt angle occurred at $\xi_{e}=45^{\circ} \pm 1^{\circ}$ rather than at $\xi_{e}=50.72^{\circ}$ as predicted by Eq. (39). This difference was addressed in Ref. 23 and is due to the fact that the rod cross section is slightly elliptical, having a majoraxis-to-minor axis ratio of $b / a=1.04$. The correction to $\xi_{e}$ on account of the elliptical cross section was derived in Ref. 23 and gave $\xi_{\theta}=45.68^{\circ}$, in agreement with the experimental observations. The parallel shadow bands and the weak diffraction rings evident in Fig. 8 are due to small local inhomogeneities in the glass. They were not apparent in our initial visual observations and were recorded only in the time exposure photographs of Figs. $8(\mathrm{a})-8(\mathrm{~d})$. These photograph correspond to $12^{\circ}$ below $\xi_{e}, 5^{\circ}$ below $\xi_{e}$, at $\xi_{e}$, and $5^{\circ}$ above $\xi_{e}$, respectively, and are to be qualitatively compared with Figs. 6(a), 6(b), 6(c), and $6(\mathrm{~d})$, respectively, which correspond to similar tilt angles below and above $\xi_{e}$. The slightly elliptical cross section of the rod and the large difference between the cylinder radii in Figs. 6 and 8 (i.e., $0.1 \mathrm{~mm}$ in Fig. 6 and $7.6 \mathrm{~mm}$ in Fig. 8) preclude a quantitative comparison. In Figs. 8(a) and 8(b), the two rainbow branches and a few of their supernumeraries are visible. In Fig. 8(c) the rain- 
bow scattering angle is $180^{\circ}$. The single rainbow peakbreaks into two closely spaced peaks near the bottom of the photograph because the film holder was not exactly parallel to the scattered conical wave front. Figure $8(\mathrm{~d})$ shows the interference pattern of the backscattered $p$ $=0, p=2$, and perhaps $p=4$ rays.

Last, a black card with a narrow slit cut into it was placed at the location $\mathrm{AA}^{\prime}$ in Fig. 7 so as to block most of the light rays illuminating the rod. When the slit was oriented nearly perpendicularly to the rod axis, rays with different impact parameters struck the rod at different heights. Photographs of the scattered intensity produced by this narrow-band illumination are shown in Figs. 9(a), 9 (b), and 9 (c) for $\xi=40^{\circ}, 45^{\circ}$, and $50^{\circ}$, respectively. The scattered intensity in these photographs traces out the dependence of the scattering angle $\theta$ as a function of the ray impact parameter. These figures are to be compared with the ray predictions of Figs. 5(a), 5(b), and 5(c), respectively, which correspond to similar angles below and above $\xi_{e}$. For $\xi<\xi_{e}$ two rainbow-producing extrema in the scattering angle are evident. For $\xi=\xi_{e}$ the scattering angle critical point is apparent, and for $\xi>\xi_{e}$ no rainbow-producing extrema occur. Although glare point coalescence at caustic transitions has been observed previously, ${ }^{26,27}$ Fig. 9 is the first direct observation, to our knowledge, of the coalescence of scattering angle extrema at a caustic transition.

\section{CONCLUSIONS}

Obtaining the Debye series for electromagnetic scattering by a particle with a high degree of symmetry, such as a circular cylinder at diagonal incidence, does not help one to compute the scattered intensity any better, since the full partial-wave scattering amplitudes are known exactly (see, however, Ref. 19). But it helps one immeasurably in trying to understand what the various features of the scattered intensity mean physically. With the help of the Debye series, wave scattering may be viewed as the sum of the physical processes of diffraction, reflection, transmission, and transmission following a number of internal reflections. For the diagonal incidence/cylinder geometry, diffraction is polarization preserving, while each transmission and each reflection have both polarizationpreserving and cross-polarized components. Our view of the first-order rainbow extinction transition is distorted and partially obscured, either experimentally or computationally, by its interference with specularly backreflected light. The computed one-internal-reflection intensity obtained by employing only the $p=2$ terms of the Debye series removes the distortion and permits an unimpeded view of the rainbow extinction transition in wave theory. The transition occurs as the two scattering angle extrema coalesce and vanish, as is the case for optical caustic transitions in general. ${ }^{28}$ It was especially pleasing to observe this coalescence directly when the black card containing the narrow slit was placed in front of our glass rod, which was then tilted and swept through the transition. The results presented here renew our conviction that the De- bye series is an extremely useful tool for obtaining an intuitive understanding of the details of electromagnetic scattering.

\section{REFERENCES}

1. J. R. Wait, "Scattering of a plane wave from a circular dielectric cylinder at oblique incidence," Can. J. Phys. 33, 189-195 (1955).

2. J. R. Reitz, F. J. Milford, and R. W. Christy, Foundations of Electromagnetic Theory, 3rd ed. (Addison-Wesley, Reading, Mass., 1979), Sec. 17.5, pp. 372-378.

3. M. Kerker, D. Cooke, W. A. Farone, and R. A. Jacobsen, "Electromagnetic scattering from an infinite circular cylinder at oblique incidence. I. Radiance functions for $m$ = 1.46," J. Opt. Soc. Am. 56, 487-491 (1966).

4. A. Cohen and C. Acquista, "Light scattering by tilted cylinders: properties of the partial wave coefficients," J. Opt. Soc. Am. 72, 531-534 (1982).

5. Y. Takano and M. Tanaka, "Phase matrix and cross sections for single scattering by circular cylinders: a comparison of ray optics and wave theory," Appl. Opt. 19, 27812793 (1980).

6. H. C. van de Hulst, Light Scattering by Small Particles (Dover, New York, 1981), Sec. 12.33, pp. 210-214.

7. K. W. Ford and J. A. Wheeler, "Semiclassical description of scattering," Ann. Phys. (N.Y.) 7, 259-286 (1959).

8. H. M. Nussenzveig, "High-frequency scattering by a transparent sphere. I. Direct reflection and transmission," J. Math. Phys. (N.Y.) 10, 82-124 (1969).

9. M. V. Berry and K. E. Mount, "Semiclassical approximations in wave mechanics," Rep. Prog. Phys. 35, 315-397 (1972), Sec. 6.

10. J. A. Lock, "Scattering of a diagonally incident focused Gaussian beam by an infinitely long homogeneous circular cylinder," J. Opt. Soc. Am. A 14, 640-652 (1997).

11. J. A. Lock, "Morphology-dependent resonances of an infinitely long circular cylinder illuminated by a diagonally incident plane wave or a focused Gaussian beam,” J. Opt. Soc. Am. A 14, 653-661 (1997).

12. E. A. Hovenac and J. A. Lock, "Assessing the contributions of surface waves and complex rays to far-field Mie scattering by use of the Debye series," J. Opt. Soc. Am. A 9, 781795 (1992).

13. E. Hecht, Optics, 2nd ed. (Addison-Wesley, Reading, Mass., 1987), Sec. 4.3 , pp. 92-113.

14. R. P. Feynman and A. R. Hibbs, Quantum Mechanics and Path Integrals (McGraw-Hill, New York, 1965), pp. 20-21, $122-123$.

15. Ref. 6, Sec. $12-32$, pp. 209-210.

16. P. Debye, "Das Elektromagnetische Feld um einen Zylinder und die Theorie des Regenbogens," Phys. Z. 9, 775-778 (1908); reprinted and translated into English in P. L. Marston, ed., Geometrical Aspects of Scattering, Milestone Series Vol. MS89 (Society of Photo-Optical Instrumentation Engineers, Bellingham, Wash., 1994), pp. 198-204.

17. B. van der Pol and H. Bremmer, "The diffraction of electromagnetic waves from an electrical point source round a finite conducting sphere, with applications to radiotelegraphy and the theory of the rainbow," Philos. Mag. 24, 141176, 825-864 (1937).

18. J. A. Lock, "Cooperative effects among partial waves in Mie scattering," J. Opt. Soc. Am. A 5, 2032-2044 (1988).

19. K. A. Fuller, "Scattering of light by coated spheres," Opt. Lett. 18, 257-259 (1993).

20. J. A. Lock, J. M. Jamison, and C.-Y. Lin, "Rainbow scattering by a coated sphere," in Light and Color in the Open Air Technical Digest Vol. 13 of 1993 OSA Technical Digest Series (Optical Society of America, Washington, D.C., 1993), pp. 8-11.

21. J. A. Lock, J. M. Jamison, and C.-Y. Lin, "Rainbow scattering by a coated sphere," Appl. Opt. 33, 4677-4690, 4960 (1994). 
22. J. J. Sakurai, Advanced Quantum Mechanics (AddisonWesley, Reading, Mass., 1980), Chap. 4, pp. 179-296, especially p. 241.

23. C. L. Adler, J. A. Lock, B. R. Stone, and C. J. Garcia, "Highorder interior caustics produced in scattering of a diagonally incident plane wave by a circular cylinder," J. Opt. Soc. Am. A 14, 1305-1315 (1997).

24. C. F. Bohren and D. R. Huffman, Absorption and Scattering of Light by Small Particles (Wiley, New York, 1983), App. C.
25. H. M. Nussenzveig, "Complex angular momentum theory of the rainbow and the glory," J. Opt. Soc. Am. 69, 1068-1079, 1193-1194 (1979).

26. P. L. Marston, "Geometrical and catastrophe optics methods in scattering," Phys. Acoust. 21, 1-234 (1992), p. 92, Fig. 40.

27. R. A. R. Tricker, Introduction to Meteorological Optics (American Elsevier, New York, 1970), p. 45, plate III.

28. M. V. Berry and C. Upstill, "Catastrophe optics: morphologies of caustics and their diffraction patterns," Prog. Opt. 18, 257-346 (1980). 